\title{
Basic Brackets of a 2D Model for the Hodge Theory without its Canonical Conjugate Momenta
}

\author{
R. Kumar $(a) *$, S. Gupta ${ }^{(a)}$ 丹, R. P. Malik ${ }^{(a, b)}$ \\ (a) Physics Department, Centre of Advanced Studies, \\ Banaras Hindu University, Varanasi - 221 005, (U.P.), India \\ (b) DST Centre for Interdisciplinary Mathematical Sciences, \\ Faculty of Science, Banaras Hindu University, Varanasi - 221 005, India \\ e-mails: rohit.kumar@bose.res.in; saurabh@if.usp.br; rpmalik1995@gmail.com
}

\begin{abstract}
We deduce the canonical brackets for a two (1+1)-dimensional (2D) free Abelian 1-form gauge theory by exploiting the beauty and strength of the continuous symmetries of a Becchi-Rouet-Stora-Tyutin (BRST) invariant Lagrangian density that respects, in totality, six continuous symmetries. These symmetries entail upon this model to become a field theoretic example of Hodge theory. Taken together, these symmetries enforce the existence of exactly the same canonical brackets amongst the creation and annihilation operators that are found to exist within the standard canonical quantization scheme. These creation and annihilation operators appear in the normal mode expansion of the basic fields of this theory. In other words, we provide an alternative to the canonical method of quantization for our present model of Hodge theory where the continuous internal symmetries play a decisive role. We conjecture that our method of quantization is valid for a class of field theories that are tractable physical examples for the Hodge theory. This statement is true in any arbitrary dimension of spacetime.
\end{abstract}

PACS: 11.15.-q, 03.70.+k

Keywords: 2D free Abelian 1-form gauge theory; symmetry principles; continuous internal symmetries; canonical (anti)commutators; creation and annihilation operators; conserved charges as generators; Hodge theory

*Present address: S. N. Bose National Centre for Basic Sciences, Block JD, Sector III,

Salt Lake, Kolkata-700098, India

†Present address: Instituto de Física, Universidade de São Paulo, C. Postal 66318,

05314-970 São Paulo, SP, Brazil 


\section{Introduction}

Symmetry principles, through the ages, have helped physicists to unravel some of the deepest mysteries of the nature. It is well-known, for instance, that the symmetries govern interactions [1]. They lead to the conservation laws in the realm of classical and quantum systems and dictate selection rules in the context of the latter. In our present investigation, we establish that the continuous symmetries lead to the derivation of basic (anti)commutators that are at the heart of the covariant canonical quantization of a gauge theory within the framework of Becchi-Rouet-Stora-Tyutin (BRST) formalism.

The (anti)BRST symmetries and the related geometry (see. e.g. [2-8]), even though discovered (and reformulated) nearly four decades ago, are still relevant in the context of superstring theories, supersymmetric (SUSY) gauge theories, perturbative quantum gravity, topological field theories, higher $p$-form $(p=2,3 \ldots)$ gauge theories (see, e.g. [9-20]). The deep connections of this technique with the ideas of superspace formalism $[19,20]$, its interpretation in the language of differential geometry; its very useful application in the quantization of gauge and reparametrization invariant theories, etc., have always kept this formalism at the forefront of research in theoretical high energy physics. In our present endeavor, we touch upon a novel aspect of this technique where it provides an alternative to the mathematical definition of canonical conjugate momenta for the canonical quantization of a 2D free Abelian gauge field theory which belongs to a class of field theories which are models for the Hodge theory.

In the canonical method of quantization (for a given field theoretic model), a triplet of central ideas are exploited. These are the usual spin-statistics relations, definition of the canonical conjugate momenta and normal ordering. First, using the usual spin-statistics relation, we distinguish between the bosonic and fermionic field variables. Second, we compute the canonical conjugate momenta corresponding to the field variables from the Lagrangian density of a given field theoretic system and define the (graded) Poisson brackets. The latter are upgraded to the (anti)commutators at the quantum level. Ultimately, in terms of the normal mode expansions of the basic fields and their corresponding momenta, the above (anti)commutators turn into the (anti)commutators amongst the creation and annihilation operators and, then, the basic brackets of the theory ensue. The physical quantities of interest (e.g. Hamiltonian, conserved charges, etc.), expressed in terms of the creation and annihilation operators are, finally, normal ordered to make physical sense.

In our present investigation, we shall utilize the virtues of the usual spin-statistic relations and normal ordering but we shall not take the help of the definition of canonical conjugate momenta in our central goal of obtaining the correct basic brackets amongst the creation and annihilation operators of our present field theoretic model for the Hodge theory (i.e. 2D free Abelian 1-form gauge theory) within the framework of BRST formalism. Rather, we shall exploit the beauty and strength of the continuous symmetries (and their generators) to obtain the correct (anti)commutators amongst the creation and annihilation operators of our present theory which incorporates fermionic as well as bosonic field operators. In fact, it is the strength of all the six continuous symmetry transformations for this 
Hodge theory that entails upon the basic (anti)commutators to emerge in a very natural fashion.

In our present paper, we demonstrate that the (anti-)BRST, (anti-)co-BRST, ghost and a bosonic symmetry transformations of a free 2D Abelian 1-form gauge theory (which happens to be a field theoretic model for the Hodge theory [2]) imply the existence of the canonical brackets that are required for the covariant canonical quantization of the above theory within the framework of BRST formalism [3-6]. We emphasize that all the above symmetries, taken together, lead to the derivation of the one and the same canonical brackets [see, equation (17) below] that are also derived by exploiting the usual canonical method from the Lagrangian density (see, Sec. 5 below). Thus, it is clear that the multi-faceted usefulness of the continuous symmetries enforce the existence of covariant canonical brackets, too, for a class of field theories that turn out to be the models for the Hodge theory.

The prime factors that have propelled us to pursue our present investigation are as follows. First and foremost, it is very exciting to note that a set of continuous symmetries, in some sense, dictate the basic canonical brackets of a given class of theories that are models for the Hodge theory. Second, our present work has the potential to be generalized to the case of 4D free Abelian 2-form gauge theory which is also endowed with a set of six continuous symmetry transformations [21]. Third, the derivation of the basic canonical brackets from the symmetry consideration (even though algebraically more involved) is more beautiful than the usual derivation of the same by exploiting the mathematical definition of the canonical momenta from a given Lagrangian density. Finally, our present work adds yet another glittering feather in the already shinning crown of the theoretical versatility of symmetry principles (because it is the strength of the latter that the mathematical definition of canonical conjugate momenta has been replaced by the ideas of continuous symmetries and their generators as the Noether conserved charges).

The contents of our paper are organized as follows. In Sec. 2, we discuss various continuous symmetry properties of the Lagrangian density of the 2D free Abelian 1-form gauge theory. Our Sec. 3 is devoted to the derivation of conserved charges which, in turn, are expressed in terms of the creation and annihilation operators. The derivation of the canonical (anti)commutators by exploiting the basic tenets of symmetry principles has been carried out in Sec. 4. Our Sec. 5 focuses on the derivation of the canonical brackets from the standard canonical method of quantization applied to the Lagrangian density of the theory. Finally, in Sec. 6, we make some concluding remarks and discuss the uniqueness of the basic brackets in our Appendix A.

Notations and convention: We adopt here the convention such that the $(1+1)-$ dimensional (2D) flat Minkowskian metric $\eta_{\mu \nu}$ is endowed with signatures $(+1,-1)$ and $P \cdot Q=\eta_{\mu \nu} P^{\mu} Q^{\nu}=P_{0} Q_{0}-P_{i} Q_{i}$ is the dot product between two non-null vectors $P_{\mu}$ and $Q_{\mu}$. Here the Greek indices $\mu, \nu, \ldots=0,1$ and Latin indices $i, j, \ldots=1$. The 2D $F_{\mu \nu}$ has only electric field as its non-vanishing component (i.e. $F_{01}=-\varepsilon^{\mu \nu} \partial_{\mu} A_{\nu}=E$ ). We take 2D Levi-Civita tensor $\left(\varepsilon_{\mu \nu}\right)$ with the choice $\varepsilon_{01}=+1=-\varepsilon^{01}$ and it obeys $\varepsilon_{\mu \nu} \varepsilon^{\mu \nu}=-2$ !, $\varepsilon_{\mu \nu} \varepsilon^{\nu \lambda}=\delta_{\mu}^{\lambda}$. We also have the d'Alembertian operator as $\square=\partial_{0}^{2}-\partial_{1}^{2}$. 


\section{Lagrangian formalism: continuous symmetries}

We begin with the (anti-)BRST invariant Lagrangian density for a free 2D Abelian 1-form gauge theory in the Feynman gauge:

$$
\begin{aligned}
\mathcal{L}_{b} & =-\frac{1}{4} F^{\mu \nu} F_{\mu \nu}-\frac{1}{2}(\partial \cdot A)^{2}-i \partial_{\mu} \bar{C} \partial^{\mu} C \\
& \equiv \frac{1}{2} E^{2}-\frac{1}{2}(\partial \cdot A)^{2}-i \partial_{\mu} \bar{C} \partial^{\mu} C
\end{aligned}
$$

where $F_{\mu \nu}=\partial_{\mu} A_{\nu}-\partial_{\nu} A_{\mu}$ is the curvature tensor derived from the 2-form $F^{(2)}=d A^{(1)} \equiv$ $\frac{1}{2}\left(d x^{\mu} \wedge d x^{\nu}\right) F_{\mu \nu}$. Here $d=d x^{\mu} \partial_{\mu}$ (with $\left.d^{2}=0\right)$ is the exterior derivative and connection 1-form $A^{(1)}=d x^{\mu} A_{\mu}$ defines the vector potential $A_{\mu}$. The gauge-fixing term $\left[-\frac{1}{2}(\partial \cdot A)^{2}\right]$ owes its origin to the co-exterior derivative $\delta=-* d *$ because $\delta A^{(1)} \equiv-* d * A^{(1)}=+(\partial$. $A)$ where the $(*)$ operation is the Hodge duality (defined on the 2D Minkowski spacetime manifold). The fermionic (anti-)ghost fields $(\bar{C}) C$ (with $C^{2}=\bar{C}^{2}=0, C \bar{C}+\bar{C} C=0$, etc.) are required for the validity of the unitarity within the framework of BRST formalism.

The Lagrangian density (1) respects the on-shell (i.e. $\square C=0, \square \bar{C}=0$ ) nilpotent (i.e. $\left.s_{(a) b}^{2}=0\right)\left(\right.$ anti-)BRST symmetry transformations $\left(s_{(a) b}\right)$ as

$$
\begin{aligned}
s_{b} A_{\mu}=\partial_{\mu} C, & s_{b} C=0, & s_{b} \bar{C}=-i(\partial \cdot A), & s_{b} E=0, \\
s_{a b} A_{\mu}=\partial_{\mu} \bar{C}, & s_{a b} \bar{C}=0, & s_{a b} C=+i(\partial \cdot A), & s_{a b} E=0,
\end{aligned}
$$

where the physical (gauge-invariant) electric field (E), owing its origin to exterior derivative $d=d x^{\mu} \partial_{\mu}$, remains invariant under the (anti-)BRST symmetry transformations (2). We have the following on-shell $(\square C=0, \square \bar{C}=0)$ nilpotent $\left(s_{(a) d}^{2}=0\right)$, continuous and infinitesimal (anti-)co-BRST symmetry transformations $s_{(a) d}$ (see, e.g. Ref. [22] and references therein)

$$
\begin{aligned}
& s_{d} A_{\mu}=-\varepsilon_{\mu \nu} \partial^{\nu} \bar{C}, \quad s_{d} \bar{C}=0, \quad s_{d} C=-i E, \quad s_{d}(\partial \cdot A)=0, \\
& s_{a d} A_{\mu}=-\varepsilon_{\mu \nu} \partial^{\nu} C, \quad s_{a d} C=0, \quad s_{a d} \bar{C}=+i E, \quad s_{a d}(\partial \cdot A)=0,
\end{aligned}
$$

that leave the Lagrangian density (1) quasi-invariant [22]. It should be noted here that the gauge-fixing term $(\partial \cdot A)$, owing its origin to the co-exterior derivative, remains invariant under the (anti-)co-BRST transformations.

A bosonic symmetry $\left(s_{\omega}\right)$ (as the anticommutator $\left\{s_{b}, s_{d}\right\} \equiv-\left\{s_{a b}, s_{a d}\right\}=s_{\omega}$ ) leads to the following transformations $[22]$

$$
\begin{aligned}
& s_{\omega} A_{\mu}=\partial_{\mu} E-\varepsilon_{\mu \nu} \partial^{\nu}(\partial \cdot A), \quad s_{\omega} E=\square(\partial \cdot A), \\
& s_{\omega} C=0, \quad s_{\omega} \bar{C}=0, \quad s_{\omega}(\partial \cdot A)=\square E,
\end{aligned}
$$

under which the Lagrangian density (1) transforms to a total spacetime derivative (see, e.g. Ref. [22]). Furthermore, we have an infinitesimal ghost symmetry transformation $\left(s_{g}\right)$ in the theory, namely;

$$
s_{g} A_{\mu}=0, \quad s_{g} C=+C, \quad s_{g} \bar{C}=-\bar{C}, \quad s_{g} E=0, \quad s_{g}(\partial \cdot A)=0,
$$


which is derived from the scale transformations $\left(C \rightarrow e^{+\Lambda} C, \bar{C} \rightarrow e^{-\Lambda} \bar{C}, A_{\mu} \rightarrow e^{0} A_{\mu}\right)$ where the infinitesimal scale parameter $\Lambda$ is spacetime independent and, for the sake of brevity, it has been set equal to one in the above transformations. It is, thus, crystal clear that we have a set of six continuous symmetries in the theory. Two of these (i.e. $s_{g}, s_{\omega}$ ) are bosonic in nature and rest of them (i.e. $\left.s_{(a) b}, s_{(a) d}\right)$ are fermionic $\left(s_{(a) b}^{2}=0, s_{(a) d}^{2}=0\right)$. The latter property is nothing but the nilpotency of order two that is associated with the (anti-)BRST and (anti-)co-BRST symmetry transformations.

\section{Conserved charges in terms of the creation and an- nihilation operators: normal ordered expressions}

The continuous symmetry transformations, according to Noether's theorem, lead to the derivation of the conserved currents. These, in turn, provide us the expressions for the conserved charges (i.e. $\left.Q_{r}=\int d x J_{r}^{0}, r=b, a b, d, a d, g, \omega\right)$. These charges for our present theory are (see, e.g. Ref. [22]).

$$
\begin{array}{rlrl}
Q_{b} & =\int d x\left[\partial_{0}(\partial \cdot A) C-(\partial \cdot A) \dot{C}\right], & & Q_{a b}=\int d x\left[\partial_{0}(\partial \cdot A) \bar{C}-(\partial \cdot A) \dot{\bar{C}}\right], \\
Q_{d}=\int d x[E \dot{\bar{C}}-\dot{E} \bar{C}], & & Q_{a d}=\int d x[E \dot{C}-\dot{E} C], \\
Q_{\omega}=\int d x\left[\partial_{0}(\partial \cdot A) E-\dot{E}(\partial \cdot A)\right], & Q_{g}=i \int d x[C \dot{\bar{C}}+\bar{C} \dot{C}],
\end{array}
$$

where the dot, on a generic field $\Phi$, denotes the time derivative [i.e. $\dot{\Phi}=(\partial \Phi) /(\partial t)]$. We lay emphasis on the fact that these conserved charges have been computed from the Noether conserved current where the definition of canonical momentum plays no role at all. In fact, it is the action principle (i.e. $\delta S=0$ ) that plays a decisive role in the above derivations.

It is evident, from the Lagrangian density (1), that the basic fields of the theory satisfy the following Euler-Lagrange equations of motion:

$$
\square A_{\mu}=0, \quad \square C=0, \quad \square \bar{C}=0 .
$$

The normal mode expansions of these fields, in the phase space of our present theory, are listed below (see, e.g. Ref. [23])

$$
\begin{aligned}
A_{\mu}(x, t) & =\int \frac{d k}{\sqrt{2 \pi 2 k_{0}}}\left[a_{\mu}(k) e^{+i k \cdot x}+a_{\mu}^{\dagger}(k) e^{-i k \cdot x}\right], \\
C(x, t) & =\int \frac{d k}{\sqrt{2 \pi 2 k_{0}}}\left[c(k) e^{+i k \cdot x}+c^{\dagger}(k) e^{-i k \cdot x}\right], \\
\bar{C}(x, t) & =\int \frac{d k}{\sqrt{2 \pi 2 k_{0}}}\left[\bar{c}(k) e^{+i k \cdot x}+\bar{c}^{\dagger}(k) e^{-i k \cdot x}\right],
\end{aligned}
$$

where 2-vector $k_{\mu}=\left(k_{0}, k_{1}=k\right)$ is the momentum vector and $a_{\mu}^{\dagger}(k), c^{\dagger}(k)$ and $\bar{c}^{\dagger}(k)$ are the creation operators for a photon, a ghost and an anti-ghost quanta, respectively. 
The non-dagger operators $a_{\mu}(k), c(k)$ and $\bar{c}(k)$ stand for the corresponding annihilation operators for a single quantum.

Plugging in these expansions in the expressions for the charges in (6), we obtain the following

$$
\begin{aligned}
Q_{b} & =-\int d k k^{\mu}\left[a_{\mu}^{\dagger}(k) c(k)+c^{\dagger}(k) a_{\mu}(k)\right], \\
Q_{a b} & =-\int d k k^{\mu}\left[a_{\mu}^{\dagger}(k) \bar{c}(k)+\bar{c}^{\dagger}(k) a_{\mu}(k)\right], \\
Q_{d} & =-\int d k \varepsilon^{\mu \nu} k_{\mu}\left[\bar{c}^{\dagger}(k) a_{\nu}(k)+a_{\nu}^{\dagger}(k) \bar{c}(k)\right], \\
Q_{a d} & =-\int d k \varepsilon^{\mu \nu} k_{\mu}\left[c^{\dagger}(k) a_{\nu}(k)+a_{\nu}^{\dagger}(k) c(k)\right], \\
Q_{g} & =-\int d k\left[\bar{c}^{\dagger}(k) c(k)+c^{\dagger}(k) \bar{c}(k)\right], \\
Q_{\omega} & =i \int d k \varepsilon^{\mu \nu} k_{\mu} k^{\rho}\left[a_{\rho}^{\dagger}(k) a_{\nu}(k)-a_{\nu}^{\dagger}(k) a_{\rho}(k)\right] \\
& \equiv i \int d k k^{2} \varepsilon^{\mu \nu} a_{\mu}^{\dagger}(k) a_{\nu}(k),
\end{aligned}
$$

where the normal ordering has been taken into account so that all the creation operators are kept towards the left. This ordering renders the above charges physically sensible. In the above, we have taken into account the expression for the Dirac $\delta$-function as: $\delta\left(k-k^{\prime}\right)=$ $(1 / 2 \pi) \int d x e^{ \pm\left(k-k^{\prime}\right) \cdot x}$ in the explicit computations of charges in equation (9). Thus, we have already exploited one of the key requirements of the quantization scheme, we have proposed to follow.

\section{Canonical brackets: symmetry considerations}

According to the common folklore in quantum field theory, the conserved charges (6) (that are derived due to the presence of continuous symmetries in the theory) generate the continuous symmetry transformations, as

$$
s_{r} \Phi= \pm i\left[\Phi, Q_{r}\right]_{ \pm}, \quad r=b, a b, d, a d, \omega, g,
$$

where $\Phi$ is the generic field of the theory and $Q_{r}$ are the conserved charges of the theory $[\mathrm{cf} .(6)]$. The $( \pm)$ signs, as a subscript on the square bracket, correspond to the (anti)commutators for the generic field $\Phi$ being fermionic (bosonic) in nature. Obviously, we have used here the usual spin-statistics relations to differentiate between the bosonic and fermionic fields. Quantum mechanically, this implies the use of suitable brackets [i.e. (anti)commutators] for the quantization scheme. It should be noted that, in our present 2D theory, there is no concept of "spin" because the Pauli-Lubanski vector cannot be defined in 2D. Thus, we have not used the phrase "spin-statistics theorem" which is valid in 4D theory. The $( \pm)$ signs in front of the expression on the r.h.s. (i.e. $\left.\pm i\left[\Phi, Q_{r}\right]_{ \pm}\right)$need explanation. The pertinent points regarding the choice of a specific sign (in front of the bracket) are: 
i. for $s_{r}=s_{b}, s_{a b}, s_{d}, s_{a d}$, only the negative sign would be taken into account (i.e. $s_{b} A_{\mu}=$ $-i\left[A_{\mu}, Q_{b}\right], s_{b} \bar{C}=-i\left\{\bar{C}, Q_{b}\right\}$, etc.), and

ii. for $s_{r}=s_{g}, s_{\omega}$, the negative sign would be taken into account for the bosonic field and the positive sign would be chosen for the fermionic field (e.g. $s_{g} A_{\mu}=$ $-i\left[A_{\mu}, Q_{g}\right], s_{g} C=+i\left[C, Q_{g}\right], s_{g} \bar{C}=+i\left[\bar{C}, Q_{g}\right]$, etc. $)$.

At this juncture, let us take an example (i.e. $s_{b} A_{\mu}=\partial_{\mu} C$ ) to make it clear that the symmetry principles dictate the structure of the canonical brackets. Mathematically, this symmetry transformation can be expressed as [23]

$$
s_{b} A_{\mu}=-i\left[A_{\mu}, Q_{b}\right]=\partial_{\mu} C .
$$

Now taking the normal mode expansions for $A_{\mu}$ and $C$ [from equation (8)], it is clear that we have the following relationships

$$
\left[Q_{b}, a_{\mu}(k)\right]=k_{\mu} c(k), \quad\left[Q_{b}, a_{\mu}^{\dagger}(k)\right]=-k_{\mu} c^{\dagger}(k) .
$$

Plugging in the expression for $Q_{b}$ in terms of the creation and annihilation operators [cf. (9)], we obtain

$$
\begin{aligned}
& {\left[a_{\mu}(k), a_{\nu}^{\dagger}\left(k^{\prime}\right)\right]=\eta_{\mu \nu} \delta\left(k-k^{\prime}\right),} \\
& {\left[a_{\mu}^{\dagger}(k), a_{\nu}^{\dagger}\left(k^{\prime}\right)\right]=0, \quad\left[a_{\mu}^{\dagger}(k), c\left(k^{\prime}\right)\right]=0, \quad\left[a_{\mu}^{\dagger}(k), c^{\dagger}\left(k^{\prime}\right)\right]=0,} \\
& {\left[a_{\mu}(k), a_{\nu}\left(k^{\prime}\right)\right]=0, \quad\left[a_{\mu}(k), c^{\dagger}\left(k^{\prime}\right)\right]=0, \quad\left[a_{\mu}(k), c\left(k^{\prime}\right)\right]=0 .}
\end{aligned}
$$

In exactly similar fashion, the following BRST symmetry transformations

$$
\begin{aligned}
& s_{b} C=-i\left\{C, Q_{b}\right\}=0 \Longrightarrow\left\{Q_{b}, c(k)\right\}=0, \quad\left\{Q_{b}, c^{\dagger}(k)\right\}=0, \\
& s_{b} \bar{C}=-i\left\{\bar{C}, Q_{b}\right\}=-i(\partial \cdot A) \Longrightarrow \\
& \left\{Q_{b}, \bar{c}(k)\right\}=+i k^{\mu} a_{\mu}(k), \quad\left\{Q_{b}, \bar{c}^{\dagger}(k)\right\}=-i k^{\mu} a_{\mu}^{\dagger}(k),
\end{aligned}
$$

lead to the derivation of the following brackets

$$
\begin{aligned}
& \left\{c^{\dagger}(k), \bar{c}\left(k^{\prime}\right)\right\}=-i \delta\left(k-k^{\prime}\right), \quad\left\{c(k), \bar{c}\left(k^{\prime}\right)\right\}=0, \\
& \left\{c(k), \bar{c}^{\dagger}\left(k^{\prime}\right)\right\}=+i \delta\left(k-k^{\prime}\right), \quad\left\{c^{\dagger}(k), \bar{c}^{\dagger}\left(k^{\prime}\right)\right\}=0, \\
& {\left[a_{\mu}^{\dagger}(k), \bar{c}\left(k^{\prime}\right)\right]=0, \quad\left[a_{\mu}(k), \bar{c}\left(k^{\prime}\right)\right]=0, \quad\left[a_{\mu}^{\dagger}(k), \bar{c}^{\dagger}\left(k^{\prime}\right)\right]=0,} \\
& {\left[a_{\mu}(k), \bar{c}^{\dagger}\left(k^{\prime}\right)\right]=0, \quad\left[a_{\mu}^{\dagger}(k), c\left(k^{\prime}\right)\right]=0, \quad\left[a_{\mu}(k), c\left(k^{\prime}\right)\right]=0 .}
\end{aligned}
$$

It is worthwhile to point out that the following statements are true, namely;

i. the above brackets have been derived by taking into account (see, e.g. Ref. [23] for details) only the on-shell nilpotent BRST symmetry transformations [i.e. $s_{b} A_{\mu}=$ $\left.\partial_{\mu} C, s_{b} C=0, s_{b} \bar{C}=-i(\partial \cdot A)\right]$, and

ii. the expressions on the r.h.s. of equations (12) and (14) enforce, in a definite manner, the choice of the (anti)commutators in (13) and (15) when we use the expression for $Q_{b}$ from (9). 
We would like to emphasize that the above exercise can be performed with all the six conserved charges listed in (6). The relevant (anti)commutators, emerging out from this algebraic exercise, are as follows

$$
\begin{array}{ll}
{\left[Q_{a b}, a_{\mu}(k)\right]=+k_{\mu} \bar{c}(k),} & \left\{Q_{a b}, \bar{c}(k)\right\}=0, \\
{\left[Q_{a b}, a_{\mu}^{\dagger}(k)\right]=-k_{\mu} \bar{c}^{\dagger}(k),} & \left\{Q_{a b}, \bar{c}^{\dagger}(k)\right\}=0, \\
\left\{Q_{a b}, c(k)\right\}=-i k^{\mu} a_{\mu}(k), & \left\{Q_{d}, \bar{c}(k)\right\}=0, \\
\left\{Q_{a b}, c^{\dagger}(k)\right\}=+i k^{\mu} a_{\mu}^{\dagger}(k), \quad & \left\{Q_{d}, \bar{c}^{\dagger}(k)\right\}=0, \\
{\left[Q_{d}, a_{\mu}(k)\right]=-\varepsilon_{\mu \nu} k^{\nu} \bar{c}(k),} & \left\{Q_{a d}, c(k)\right\}=0, \\
{\left[Q_{d}, a_{\mu}^{\dagger}(k)\right]=+\varepsilon_{\mu \nu} k^{\nu} \bar{c}^{\dagger}(k),} & \left\{Q_{a d}, c^{\dagger}(k)\right\}=0, \\
\left\{Q_{d}, c(k)\right\}=-i \varepsilon^{\mu \nu} k_{\mu} a_{\nu}(k), & {\left[Q_{g}, a_{\mu}(k)\right]=0,} \\
\left\{Q_{d}, c^{\dagger}(k)\right\}=+i \varepsilon^{\mu \nu} k_{\mu} a_{\nu}^{\dagger}(k), & {\left[Q_{g}, a_{\mu}^{\dagger}(k)\right]=0,} \\
{\left[Q_{a d}, a_{\mu}(k)\right]=-\varepsilon_{\mu \nu} k^{\nu} c(k),} & {\left[Q_{g}, \bar{c}^{\dagger}(k)\right]=-i \bar{c}^{\dagger}(k),} \\
{\left[Q_{a d}, a_{\mu}^{\dagger}(k)\right]=+\varepsilon_{\mu \nu} k^{\nu} c^{\dagger}(k),} & {\left[Q_{g}, \bar{c}(k)\right]=-i \bar{c}(k)} \\
\left\{Q_{a d}, \bar{c}(k)\right\}=+i \varepsilon^{\mu \nu} k_{\mu} a_{\nu}(k), & {\left[Q_{\omega}, \bar{c}(k)\right]=0,} \\
\left\{Q_{a d}, \bar{c}^{\dagger}(k)\right\}=-i \varepsilon^{\mu \nu} k_{\mu} a_{\nu}^{\dagger}(k), & {\left[Q_{\omega}, \bar{c}^{\dagger}(k)\right]=0,} \\
{\left[Q_{g}, c(k)\right]=+i c(k),} & {\left[Q_{g}, c^{\dagger}(k)\right]=+i c^{\dagger}(k),} \\
{\left[Q_{\omega}, a_{\mu}^{\dagger}(k)\right]=-i k^{2} \varepsilon_{\mu \nu}\left(a^{\nu}\right)^{\dagger}(k),} & {\left[Q_{\omega}, c(k)\right]=0,} \\
{\left[Q_{\omega}, a_{\mu}(k)\right]=-i k^{2} \varepsilon_{\mu \nu} a^{\nu}(k),} & {\left[Q_{\omega}, c^{\dagger}(k)\right]=0 .}
\end{array}
$$

The outcome of all the above (anti)commutators, with the help of the normal mode expansions (8) and the expressions for the charges in (9), lead to the following non-vanishing basic brackets

$$
\begin{aligned}
& {\left[a_{\mu}(k), a_{\nu}^{\dagger}\left(k^{\prime}\right)\right]=\eta_{\mu \nu} \delta\left(k-k^{\prime}\right),} \\
& \left\{c(k), \bar{c}^{\dagger}\left(k^{\prime}\right)\right\}=+i \delta\left(k-k^{\prime}\right), \\
& \left\{c^{\dagger}(k), \bar{c}\left(k^{\prime}\right)\right\}=-i \delta\left(k-k^{\prime}\right),
\end{aligned}
$$

within the framework of BRST formalism. All the rest of the (anti)commutators turn out to be zero. To summarize, we have already utilized all the ingredients of our quantization

scheme (without any use of the mathematical definition of canonically conjugate momenta anywhere in our discussions).

\section{Standard canonical method: Lagrangian formalism}

It is evident that the canonical conjugate momenta from the Lagrangian density (1), for the basic fields of the theory, are

$$
\begin{aligned}
& \Pi^{\mu}=\frac{\partial \mathcal{L}_{(b)}}{\partial\left(\partial_{0} A_{\mu}\right)}=-F^{0 \mu}-\eta^{0 \mu}(\partial \cdot A) \\
& \Pi_{C}=\frac{\partial \mathcal{L}_{(b)}}{\partial\left(\partial_{0} C\right)}=+i \dot{\bar{C}}, \quad \Pi_{\bar{C}}=\frac{\partial \mathcal{L}_{(b)}}{\partial\left(\partial_{0} \bar{C}\right)}=-i \dot{C}
\end{aligned}
$$


As a result, we have the following canonical brackets:

$$
\begin{aligned}
& {\left[A_{\mu}(x, t), \Pi_{\nu}\left(x^{\prime}, t\right)\right]=i \eta_{\mu \nu} \delta\left(x-x^{\prime}\right),} \\
& \left\{\bar{C}(x, t), \Pi_{\bar{C}}\left(x^{\prime}, t\right)\right\}=i \delta\left(x-x^{\prime}\right) \Longrightarrow\left\{\bar{C}(x, t), \dot{C}\left(x^{\prime}, t\right)\right\}=-\delta\left(x-x^{\prime}\right), \\
& \left\{C(x, t), \Pi_{C}\left(x^{\prime}, t\right)\right\}=i \delta\left(x-x^{\prime}\right) \Longrightarrow\left\{C(x, t), \dot{\bar{C}}\left(x^{\prime}, t\right)\right\}=\delta\left(x-x^{\prime}\right) .
\end{aligned}
$$

All the rest of the brackets are zero. The top entry, in the above, implies the following commutators in terms of the components of the $2 \mathrm{D}$ gauge field $A_{\mu}$ and the corresponding conjugate momenta, namely;

$$
\begin{aligned}
{\left[A_{0}(x, t),(\partial \cdot A)\left(x^{\prime}, t\right)\right] } & =-i \delta\left(x-x^{\prime}\right), \\
{\left[A_{i}(x, t), E_{j}\left(x^{\prime}, t\right)\right] } & =i \delta_{i j} \delta\left(x-x^{\prime}\right) .
\end{aligned}
$$

The above form of commutators would be useful later.

To simplify, the rest of our computations, we re-express the normal mode expansions of the basic fields [cf. (8)], as [24]

$$
\begin{aligned}
A_{\mu}(x, t) & =\int d k\left[a_{\mu}(k) f^{*}(k, x)+a_{\mu}^{\dagger}(k) f(k, x)\right], \\
C(x, t) & =\int d k\left[c(k) f^{*}(k, x)+c^{\dagger}(k) f(k, x)\right], \\
\bar{C}(x, t) & =\int d k\left[\bar{c}(k) f^{*}(k, x)+\bar{c}^{\dagger}(k) f(k, x)\right],
\end{aligned}
$$

where the new functions:

$$
f(k, x)=\frac{e^{-i k \cdot x}}{\sqrt{2 \pi 2 k_{0}}}, \quad f^{*}(k, x)=\frac{e^{i k \cdot x}}{\sqrt{2 \pi 2 k_{0}}},
$$

form an orthonormal set because they satisfy [24]

$$
\begin{aligned}
& \int d x f^{*}(k, x) i \overleftrightarrow{\partial_{0}} f\left(k^{\prime}, x\right)=\delta\left(k-k^{\prime}\right), \quad \int d x f^{*}(k, x) i \overleftrightarrow{\partial_{0}} f^{*}\left(k^{\prime}, x\right)=0 \\
& \int d x f(k, x) i \overleftrightarrow{\partial_{0}} f\left(k^{\prime}, x\right)=0
\end{aligned}
$$

where we have taken into account the following definition

$$
A \overleftrightarrow{\partial_{0}} B=A\left(\partial_{0} B\right)-\left(\partial_{0} A\right) B
$$

for the operator $\overleftrightarrow{\partial_{0}}$ between two non-zero variables $A$ and $B$. Using the above relations, it is straightforward to check that

$$
\begin{array}{rlrl}
a_{\mu}(k) & =\int d x A_{\mu}(x, t) i \overleftrightarrow{\partial_{0}} f(k, x), & & a_{\mu}^{\dagger}(k)=\int d x f^{*}(k, x) i \overleftrightarrow{\partial_{0}} A_{\mu}(x, t), \\
c^{\dagger}(k) & =\int d x f^{*}(k, x) i \overleftrightarrow{\partial_{0}} C(x, t), & \bar{c}^{\dagger}(k)=\int d x f^{*}(k, x) i \overleftrightarrow{\partial_{0}} \bar{C}(x, t), \\
c(k)=\int d x C(x, t) i \overleftrightarrow{\partial_{0}} f(k, x), & \bar{c}(k)=\int d x \bar{C}(x, t) i \overleftrightarrow{\partial_{0}} f(k, x) .
\end{array}
$$


Thus, we have expressed the creation and annihilation operators in terms of the fields and the orthonormal functions $f(k, x)$ and $f^{*}(k, x)$.

At this stage, a few comments are in order. First and foremost, it is straightforward to check that only the canonical brackets (17) survive in the explicit computation. Second, there exist six anticommutators from the four fermionic operators $c(k), c^{\dagger}(k), \bar{c}(k), \bar{c}^{\dagger}(k)$. Out of which, four would be zero because of the orthonormality relations (23) and be-

cause of the fact that $C^{2}=\bar{C}^{2}=0,\left\{C(x, t), \dot{C}\left(x^{\prime}, t\right)\right\}=0,\left\{\bar{C}(x, t), \dot{\bar{C}}\left(x^{\prime}, t\right)\right\}=0$. Third, there exist three basic commutators from $a_{\mu}(k)$ and $a_{\mu}^{\dagger}(k)$. Out of which, two would turn out to be zero because the commutation relations in (20) can be recast in the form $\left[A_{\mu}(x, t), \dot{A}_{\nu}\left(x^{\prime}, t\right)\right]=-i \eta_{\mu \nu} \delta\left(x-x^{\prime}\right)$ due to the fact that (i) $\dot{A}_{0}=(\partial \cdot A)+\partial_{i} A_{i}$ and $\dot{A}_{i}=E_{i}+\partial_{i} A_{0}$, and (ii) the spatial derivative of the gauge field $A_{\mu}$ commutes with itself.

It is straightforward to check that the canonical brackets of (19) and (20) [that are derived from the Lagrangian density (1)] lead to the derivation of the same brackets that are listed in (17). Thus, we conclude that the basic canonical brackets [cf. (17)] between the creation and annihilation operators of the bosonic and fermionic fields of the theory can be derived from (i) the continuous symmetry considerations, and (ii) by exploiting the definition of momenta from the Lagrangian density of the theory.

\section{Conclusions}

In our present investigation, the key ideas that have been exploited for the quantization scheme are the usual spin-statistics relations, normal ordering (in the expressions for the conserved charges) and the key concepts of the continuous symmetry transformations (and corresponding generators). The last ingredient of the above quantization scheme is the novel one and it differs from the standard method of canonical quantization scheme where the (graded) Poisson brackets (defined with the help of definition of the conjugate momenta) are promoted to the (anti)commutators in addition to the helps coming out from the usual spin-statistics relations and normal ordering. It is worthwhile to mention that, in a 2D Minkowski space, there is no concept of spin because the Pauli-Lubanski vector cannot be defined on a $2 \mathrm{D}$ spacetime manifold. Thus, the implication of the usual spin-statistics relation is the existence of (anti)commutators at the quantum level in our present 2D field theoretic model of Hodge theory.

One of the most beautiful observations in our present endeavor is the emergence of one and the same set of non-vanishing basic canonical brackets [cf. (17)] from all the continuous symmetry transformations present in the theory. These basic canonical brackets are found to be unique and any other alternatives/deformations to them would not work with all the continuous symmetries. Even though the continuous symmetry transformations (and corresponding generators) look completely different, the hidden basic brackets (17) [that emerge from the application of (10)] are exactly the same.

The above key observation ensures that the continuous symmetry transformations of a field theoretic model for the Hodge theory encode in their folds the basic (anti)commutators corresponding to the fermionic and bosonic fields of this theory. To the best of our knowl- 
edge, our method of derivation of the basic brackets in (17) is a novel observation in the realm of the quantization scheme of a gauge theory. It should be noted that two of us (SG and RK) have already generalized our present method of quantization to 4D free Abelian 2-form gauge theory [21] (which also happens to be a field theoretic model for Hodge theory [25]) and we have also obtained the exact basic (anti)commutators in the context of quantization scheme for a 2D interacting Abelian 1-form $U(1)$ theory where the Dirac fields are also present [26]. The latter fields couple with the $U(1)$ gauge field in a gauge invariant manner. In a very recent publication [27], we have derived the basic brackets at the level of creation and annihilation operators in the case of a toy model for the rigid rotor which is also is an example of the Hodge theory.

Our method of quantization is completely different from the derivation of canonical brackets by considering the equations of motion for the quantum mechanical system of harmonic oscillator by Wigner [28]. In our approach, we do not use the equations of motion anywhere except in the proof of nilpotency of the (anti-)BRST and (anti-)dual BRST symmetries [cf. (2) and (3)] respectively. Rather, we exploit the ideas of symmetry principle and the definition of generators in our approach. In fact, it is the existence of six continuous symmetries of our present model for the Hodge theory that entails upon the emergence of basic canonical brackets (17) uniquely at the level of creation/annihilation operators (that appear in the normal mode expansions of the fields). It would be very nice endeavor for us to apply our method to the quantization of other challenging filed theoretic models of Hodge theory in higher dimensions of spacetime. We are intensively involved, at the moment, with such kinds of problems and our results would be reported elsewhere [29].

\section{Acknowledgements}

SG and RK would like to gratefully acknowledge the financial support from CSIR and UGC, New Delhi, Government of India, respectively.

\section{Appendix A: On the uniqueness of the basic brackets}

Here we describe very briefly the uniqueness of the canonical brackets (taken as the (anti)commutation relations) amongst the creation and annihilation operators which have been derived in the equation (17). Exploiting the general definition (10) of the generator and the transformations (2) and (3), it can be checked that the following alternative (to 
(17)) (anti)commutators (with $A(k)=A^{\dagger}(k)$ as a parameter)

$$
\begin{aligned}
{\left[a_{\mu}(k), a_{\nu}^{\dagger}\left(k^{\prime}\right)\right] } & =\eta_{\mu \nu} \delta\left(k-k^{\prime}\right)+A(k) a_{\nu}^{\dagger}(k) a_{\mu}(k) \delta\left(k-k^{\prime}\right), \\
\left\{c^{\dagger}(k), \bar{c}\left(k^{\prime}\right)\right\} & =-i \delta\left(k-k^{\prime}\right)+A(k) c^{\dagger}(k) \bar{c}(k) \delta\left(k-k^{\prime}\right), \\
\left\{\bar{c}^{\dagger}(k), c\left(k^{\prime}\right)\right\} & =i \delta\left(k-k^{\prime}\right)+A(k) \bar{c}^{\dagger}(k) c(k) \delta\left(k-k^{\prime}\right), \\
{\left[c(k), a_{\mu}\left(k^{\prime}\right)\right] } & =A(k) a_{\mu}(k) c(k) \delta\left(k-k^{\prime}\right), \\
{\left[c^{\dagger}(k), a_{\mu}^{\dagger}\left(k^{\prime}\right)\right] } & =-A(k) c^{\dagger}(k) a_{\mu}^{\dagger}(k) \delta\left(k-k^{\prime}\right), \\
{\left[\bar{c}^{\dagger}(k), a_{\mu}^{\dagger}\left(k^{\prime}\right)\right] } & =-A(k) \bar{c}^{\dagger}(k) a_{\mu}^{\dagger}(k) \delta\left(k-k^{\prime}\right), \\
{\left[\bar{c}(k), a_{\mu}\left(k^{\prime}\right)\right] } & =A(k) a_{\mu}(k) \bar{c}(k) \delta\left(k-k^{\prime}\right), \\
\left\{\bar{c}^{\dagger}(k), \bar{c}\left(k^{\prime}\right)\right\} & =A(k) \bar{c}^{\dagger}(k) \bar{c}(k) \delta\left(k-k^{\prime}\right), \\
\left\{c^{\dagger}(k), c\left(k^{\prime}\right)\right\} & =A(k) c^{\dagger}(k) c(k) \delta\left(k-k^{\prime}\right), \\
\left\{c^{\dagger}(k), c\left(k^{\prime}\right)\right\} & =A(k) c^{\dagger}(k) c(k) \delta\left(k-k^{\prime}\right), \\
{\left[a_{\mu}(k), a_{\nu}\left(k^{\prime}\right)\right] } & =0, \quad\left[a_{\mu}^{\dagger}(k), a_{\nu}^{\dagger}\left(k^{\prime}\right)\right]=0, \\
{\left[c^{\dagger}(k), a_{\mu}\left(k^{\prime}\right)\right] } & =0, \quad\left[c(k), a_{\mu}^{\dagger}\left(k^{\prime}\right)\right]=0, \\
{\left[\bar{c}(k), a_{\mu}^{\dagger}\left(k^{\prime}\right)\right] } & =0, \quad\left[\bar{c}^{\dagger}(k), a_{\mu}\left(k^{\prime}\right)\right]=0, \\
\left\{\bar{c}(k), \bar{c}\left(k^{\prime}\right)\right\} & =0, \quad\left\{\bar{c}^{\dagger}(k), \bar{c}^{\dagger}\left(k^{\prime}\right)\right\}=0, \\
\left\{\bar{c}(k), c\left(k^{\prime}\right)\right\} & =0, \quad\left\{\bar{c}^{\dagger}(k), c^{\dagger}\left(k^{\prime}\right)\right\}=0, \\
\left\{c(k), c\left(k^{\prime}\right)\right\} & =0, \quad\left\{c^{\dagger}(k), c^{\dagger}\left(k^{\prime}\right)\right\}=0,
\end{aligned}
$$

are consistent with the appropriate brackets listed in (12), (14) and (16) with the nilpotent (anti-)BRST and (anti-)co-BRST charges when we exploit the analogue of (11) in a suitable fashion. In other words, as far as the nilpotent (anti-)BRST and (anti-)co-BRST symmetries are concerned, the brackets (26) are as good as the canonical brackets derived in (17). However, the brackets (26) fail miserably to be consistent with the other two conserved charges $Q_{\omega}$ and $Q_{g}$ [cf. (9)] when we exploit the equations (4), (5), (10) in the analogue of (11) for the test of consistency as well as preciseness of the brackets (26) in the derivation of the appropriate brackets [i.e. (anti)commutators] of (16).

To corroborate the above assertions, let us take a couple of explicit examples with $Q_{\omega}$ and $Q_{g}$ (which generate the bosonic and ghost transformations). For instance, the following two brackets from (16)

$$
\left[Q_{\omega}, a_{\mu}(k)\right]=-i k^{2} \varepsilon_{\mu \nu} a^{\nu}(k), \quad\left[Q_{g}, c(k)\right]=i c(k),
$$

must be satisfied by the alternative brackets given in (26). The l.h.s. of the first commutator (i.e. $\left.\left[Q_{\omega}, a_{\mu}(k)\right]=-i k^{2} \varepsilon_{\mu \nu} a^{\nu}(k)\right)$ of the above equation (27) implies the following commutator:

$$
\begin{aligned}
{\left[Q_{\omega}, a_{\mu}(k)\right] } & =i \int d k^{\prime} k^{\prime 2} \varepsilon^{\rho \nu}\left(a_{\rho}^{\dagger}\left(k^{\prime}\right)\left[a_{\nu}\left(k^{\prime}\right), a_{\mu}(k)\right]\right. \\
& \left.+\left[a_{\rho}^{\dagger}\left(k^{\prime}\right), a_{\mu}(k)\right] a_{\nu}\left(k^{\prime}\right)\right) .
\end{aligned}
$$

Using the appropriate brackets from (26), we obtain

$$
\left[Q_{\omega}, a_{\mu}(k)\right]=-i k^{2} \varepsilon_{\mu \nu} a^{\nu}(k)-i k^{2} \varepsilon_{\rho \nu} A(k)\left(a^{\rho}\right)^{\dagger}(k) a_{\mu}(k) a^{\nu}(k) .
$$


It is obvious that the r.h.s. of the above equation does not match with the required result of (27). Thus, the brackets (26) are not consistent. They can be consistent if and only if $A(k)=0$ which, ultimately, implies the uniqueness of non-vanishing basic canonical brackets (17).

To check the sanctity and preciseness of the brackets (26), now let us take the second commutator (i.e. $\left[Q_{g}, c(k)\right]=i c(k)$ ) of the above equation (27). The 1.h.s. of the commutator is as follows

$$
\begin{aligned}
{\left[Q_{g}, c(k)\right] } & =-\int d k^{\prime}\left(-\left\{\bar{c}^{\dagger}\left(k^{\prime}\right), c(k)\right\} c\left(k^{\prime}\right)+\bar{c}^{\dagger}\left(k^{\prime}\right)\left\{c\left(k^{\prime}\right), c(k)\right\}\right. \\
& \left.-\left\{c^{\dagger}\left(k^{\prime}\right), c(k)\right\} \bar{c}\left(k^{\prime}\right)+c^{\dagger}\left(k^{\prime}\right)\left\{\bar{c}\left(k^{\prime}\right), c(k)\right\}\right) .
\end{aligned}
$$

In the above equation, we express the ghost charge in terms of the creation and annihilation operators [cf, (9)] and choose the appropriate brackets from (26). It is evident that $\left\{c(k), c\left(k^{\prime}\right)\right\}=0,\left\{\bar{c}(k), c\left(k^{\prime}\right)\right\}=0[\mathrm{cf} .(26)]$. With these inputs, it can be checked that

$$
\left[Q_{g}, c(k)\right]=i c(k)+c^{\dagger}(k) A(k) c(k) \bar{c}(k) .
$$

The above equation is in conflict with the requirement of the r.h.s. of equation (27) [unless $A(k)=0]$. Thus, the (anti)commutators in (26) are not consistent in proving equation (27) in its exact form.

Ultimately, we conclude that, even though the brackets (26) are consistent with all the requirements connected with the charges $Q_{(a) b}$ and $Q_{(a) d}$, they are not consistent with charges $Q_{\omega}$ and $Q_{g}$. We wish to lay emphasis on the fact that we have taken only two brackets in equation (27). However, as it turns out, all the rest of the relevant brackets with $Q_{\omega}$ and $Q_{g}$ are found to be inconsistent. Hence the canonical (anti)commutators of equation (17) are unique in the sense that they are consistent with all the six continuous symmetries and corresponding conserved charges. In general, there might exist many kinds

of deformations like (26). However, the consistency with all the six continuous symmetries would always lead to the derivation of (17).

\section{References}

[1] C. N. Yang, Physics Today 33, 42 (1980)

[2] C. Becchi, A. Rouet and R. Stora, Phys. Lett. B 32, 344 (1974)

[3] C. Becchi, A. Rouet and R. Stora, Commun. Math. Phys. 42, 127 (1975)

[4] C. Becchi, A. Rouet and R. Stora, Ann. Phys. (N.Y.) 98, 287 (1976)

[5] I. V. Tyutin, Lebedev Institute Preprint (Report No: FIAN-39, 1975) (unpublished)

[6] T. Kugo, I. Ojima, Prog. Theor. Phys. Suppl. 66, 1 (1979)

[7] K. Nishijima, Czech. J. Phys. 46, 1 (1996) 
[8] See, e.g., L. Bonora and M. Tonin, Phys. Lett. B 98, 48 (1981)

[9] M. Hatsuda, Y. Huang and W. Siegel, JHEP 58, 0904 (2009)

[10] Mir Faizal, Phys. Lett. B 102, 748 (2015)

[11] C. R. Mafra, O. Schlotterer, S. Stieberger and D. Tsimpis, Nucl. Phys. B 359, 846 (2011)

[12] Mir Faizal, Phys. Rev. D 84, 10611 (2011)

[13] C. P. Constantinidis, F. Gieres, O. Piguet and M. S. Sarandy, JHEP 017, 0201 (2002)

[14] Mir Faizal, Found. Phys. 41, 270 (2011)

[15] Mir Faizal, Class. Quant. Grav. 29, 215009 (2012)

[16] A. S. Schwarz, Lett. Math. Phys. 2, 247 (1978)

[17] E. Witten, Nucl. Phys. B 202, 253 (1982)

[18] For review, see, e.g., D. Birmingham, M. Blau, M. Rakowski, and G. Thompson, Phys. Rep. 209, 129 (1991)

[19] R. Kumar, S. Krishna, A. Shukla and R. P. Malik, Int. J. Mod. Phys. A 29, 1450135 (2014)

[20] L. Bonora and R. P. Malik, J. Phys. A 43, 375403 (2010)

[21] S. Gupta and R. Kumar, arXiv:1105.1446 [hep-th]

[22] See, e.g., R. P. Malik, Int. J. Mod. Phys. A 22, 3521 (2007)

[23] S. Weinberg, The Quantum Theory of Fields: Modern Applications Vol.II (Cambridge University Press, Cambridge, 1996)

[24] L. H. Ryder, Quantum Field Theory (Cambridge University Press, Cambridge, 1985)

[25] S. Gupta and R. P. Malik, Eur. Phys. J. C 58, 517 (2008)

[26] R. Kumar, S. Gupta and R. P. Malik, Commun Theor. Phys. 61, 715 (2014)

[27] D. Shukla, T. Bhanja and R. P. Malik, Int. J. Mod. Phys. A 30, 1550115 (2015)

[28] E. P. Wigner, Phys. Rev. 77, 711 (1950)

[29] R. P. Malik, etal. In preparation 